\title{
miR-34a in serum is involved in mild-to- moderate COPD in women exposed to biomass smoke
}

Yadira Velasco-Torres ${ }^{1,2}$, Victor Ruiz-López ${ }^{3}$, Oliver Pérez-Bautista ${ }^{4}$, Ivette Buendía-Roldan ${ }^{5}$, Alejandra Ramírez-Venegas ${ }^{6}$, Julia Pérez-Ramos ${ }^{1}$, Ramcés Falfán-Valencia ${ }^{7}$, Carlos Ramos ${ }^{6^{*}}$ and Martha Montaño $0^{6^{*}}$ (D)

\begin{abstract}
Background: Chronic obstructive pulmonary disease (COPD) is characterized by persistent respiratory symptoms and airflow limitation that is due to airway and/or alveolar abnormalities. The main causes of COPD are Geneenvironment interactions associated with tobacco smoking (COPD-TS) and biomass smoke (COPD-BS). It is well know that microRNAs (miRNAs) participate in the control of post-transcriptional regulation and are involved in COPD-TS; nevertheless, those miRNAS are participating in the COPD-BS are unidentified. Thus, we studied which miRNAs are involved in COPD-BS (GOLD stages I-II).
\end{abstract}

Methods: In the screening phase, the profile of the miRNAs was analyzed in serum samples $(n=3)$ by means of a PCR array. Subsequently, the miRNAs were validated with RT-qPCR $(n=25)$ in the corresponding study groups. Additionally, the serum concentration of Notch1 was measured comparing COPD-BS vs COPD-TS.

Results: miR-34a was down-regulated in COPD- BS vs COPD-TS. In the other study groups, three miRNAs were differentially expressed: miR-374a was down-regulated in COPD-BS vs C, miR-191-5p was up-regulated in COPD-BS vs $\mathrm{H}-\mathrm{BS}$, and miR-21-5p was down-regulated in COPD-TS compared to the C group. Moreover, the serum concentration of Notch1, one of the targets of miR-34a, was increased in COPD-BS compared to women with COPD-TS.

Conclusions: This is the first study in patients with COPD due to biomass that demonstrates miRNA expression differences between patients. The observations support the concept that COPD by biomass has a different phenotype than COPD due to tobacco smoking, which could have important implications for the treatment of these diseases.

Keywords: Biomass smoke exposure, COPD, microRNAs, PCR arrays, RT-qPCR, Tobacco smoking

\section{Background}

Chronic obstructive pulmonary disease (COPD) is a common, preventable and treatable disease, characterized by persistent respiratory symptoms and airflow limitation. COPD is caused by exposure to noxious particles or gases [1]; tobacco smoke inhalation is a fundamental cause of COPD and affects both genders.

\footnotetext{
* Correspondence: carlos.ramos26@yahoo.com.mx;

mamora572002@yahoo.com.mx

${ }^{6}$ Laboratory of Cell Biology, Department of Research in Pulmonary Fibrosis,

Mexico City, Mexico

Full list of author information is available at the end of the article
}

Biomass smoke, such as that produced by wood combustion for cooking, is another risk factor that disproportionally affects women, particularly in low and middle-income countries [2].

Currently, the COPD phenotype by biomass is considered different from that caused by tobacco smoke. Unlike COPD caused by tobacco, biomass COPD tends to remain in GOLD I and II stages [3-6], and rarely progresses to emphysema [5]. Several hypotheses have been proposed to explain the plateau in the development of COPD by biomass. Among them, early airway remodeling is the most accepted explanation, since longitudinal studies have shown a different pattern in airway remodeling in

(C) The Author(s). 2019 Open Access This article is distributed under the terms of the Creative Commons Attribution 4.0 International License (http://creativecommons.org/licenses/by/4.0/), which permits unrestricted use, distribution, and 
these women. Still, the specific mechanisms that differentiate the phenotype of COPD by tobacco and biomass are largely unknown.

Biomass COPD is usually characterized by chronic bronchitis, persistent cough and phlegm. Recently, the role of miRNAs in the pathophysiology of COPD has been explored, increasing our understanding of their role in the development of phenotypic heterogeneity of COPD. miRNAs could help to the differences in COPD phenotypes; studies in tobacco COPD have reported differential expression of miR-20, miR-28-3p, miR-34c-5p, miR-100 and miR-7 in smokers, ex-smokers and non-smokers.

These miRNAs are involved in cancer detection, protein coding of inflammatory factors, macrophages and vascular inflammation regulators [7-9]. There are not reports regarding to determine the participation of miRNAs in COPD by biomass.

We aimed to compare the expression of microRNAs in women with COPD due to biomass and tobacco smoke, as will as in control women, and to determine and quantify the target of miRNAs that are being differentially expressed by COPD phenotypes.

\section{Methods}

\section{Study population}

A total of 125 women divided in five groups of 25 participants were recruited for the study. We included women with biomass COPD (COPD-BS), tobacco COPD (COPDTS), smokers without COPD (H-TS), biomass exposed without COPD (H-BS), and healthy female controls (C), whom had not history of exposure to TS or BS, and with absence of any other respiratory or non-respiratory disease as controls (see Fig. 1). The diagnosis of COPD was established according to the history of smoking or exposure to BS and pulmonary function tests followed the recommendations of the American Thoracic Society and European Respiratory Society [1] and using standardized references for Mexican population [10, 11]. All women with COPD had I-II GOLD stages.

Demographic, anthropometric and clinical data were collected including TS history ( $>10$ packs/year) and cumulative exposure to BS in hours/year by determining the average number of hours/day of exposure and the number of years of exposure; no patient with COPD was exposed to both factors. Wood was the only fuel used by

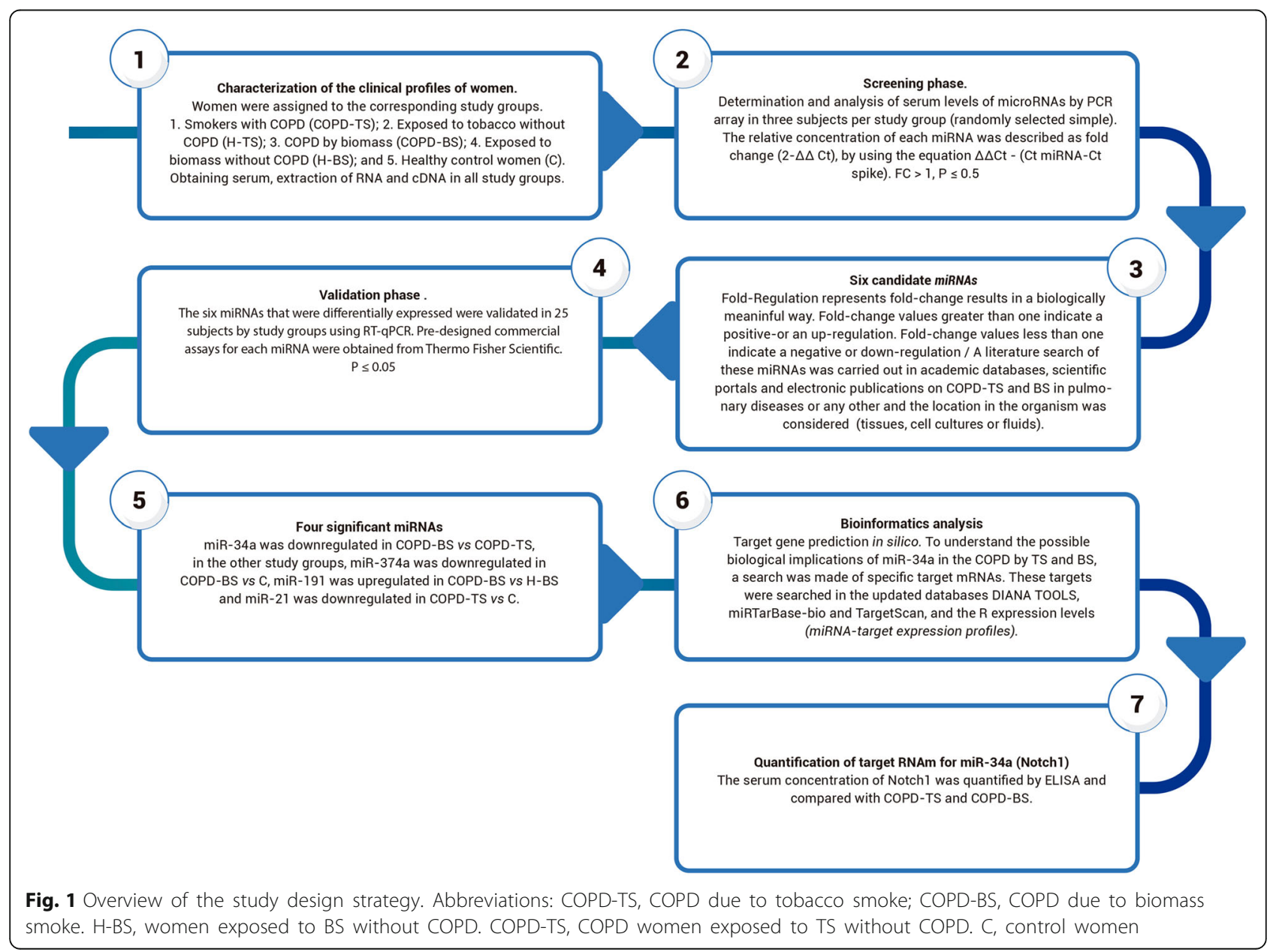


women with COPD-BS, who came from rural and suburban, low-income regions of Mexico.

\section{Blood samples}

Five $\mathrm{mL}$ of blood were collected in anticoagulant-free tubes (BD VACUTAINER, Becton, Franklin Lakes, NJ, USA), following the standard procedures at the INER, which included morning only bleeding with at least $8 \mathrm{~h}$ fasting. Samples were centrifuged at $5000 \mathrm{~g} \mathrm{X} 15 \mathrm{~min}$ and room temperature, to obtain the serum, which was kept at $-20^{\circ} \mathrm{C}$ until their analysis.

\section{Isolation of serum microRNA}

The extraction of the miRNAs was performed using the QIAGEN miRNeasy serum/plasma kit (Hilden, Germany) following the manufacturer's instructions. Aliquots of $200 \mu \mathrm{L}$ of serum were transferred into $2 \mathrm{~mL}$ tubes; QIAzol lysis reagent, $3.5 \mu \mathrm{L}$ of spike (control), $1.6 \times 10^{8}$ copies $/ \mu \mathrm{L}$, and $200 \mu \mathrm{L}$ of chloroform were added and subsequently centrifuged at $12,000 \mathrm{~g} \mathrm{X} 15 \mathrm{~min}$ at $4{ }^{\circ} \mathrm{C}$. Aqueous phase was separated and 1.5 volumes of $100 \%$ ethanol were added; later, an aliquot of $700 \mu \mathrm{L}$ was passed through a $2 \mathrm{~mL}$ RNeasy spin MinElute column and centrifuged at $8000 \mathrm{~g} \mathrm{X} 15 \mathrm{~s}$. Then, $700 \mu \mathrm{L}$ of Buffer RWT were added to the RNeasy spin MinElute column, which was centrifuged at $8000 \mathrm{~g} \mathrm{X} 15 \mathrm{~s}$ followed by the addition of $500 \mu \mathrm{L}$ of Buffer RPE. The resulting miRNA was eluted with $20 \mu \mathrm{L}$ of RNase-free water by centrifugation at $10,000 \mathrm{~g} X 1 \mathrm{~min}$. The miRNA was quantified, and integrity was assessed with the Agilent Bioanalyzer 2100 system (Agilent Technologies, Santa Clara, CA, USA).

\section{RT-qPCR Array assay in serum}

Serum miRNAs measurement was conducted in two stages: a screening stage to identify miRNAs differentially expressed in a small subsample of each participating group and a validation stage to confirm that such miRNAs were indeed different in all participants. In the screening stage we conducted a miRNAs-wide analysis, which included 96 miRNAs, using samples from three randomly selected patients from each study group. Quantitative real-time PCR (RT-qPCR) was used with the miScript miRNA PCR Array Human Serum/Plasma kit from QIAGEN (Hilden, Germany) using the StepOnePlus ${ }^{\mathrm{Th}}$ RealTime PCR System (Applied Biosystems-Real-Time PCR systems Foster City, California, USA). The data analysis was performed using software provided by the manufacturer (available at https://www.qiagen.com/ch/shop/ genes-and-pathways/data-analysis-center-overview-page/). Once miRNAs differentially expressed were identified, we implemented the validation stage in the remaining 25 participants of each group. The validation was performed by reverse transcriptase-quantitative polymerase chain reaction (RT-qPCR), obtaining the cDNA of the miRNAs extracted with the RT kit and amplified withTaqMan Universal Master Mix II with the UNG kit, all from Applied Biosystems by Thermo Fisher Scientific (USA). Pre-designed commercial assays for each miRNA were obtained from Thermo Fisher Scientific: hsa-miR-150-5p (Assay ID 000473), hsa-miR-223-3p (Assay ID 0002295), hsa-miR-191-5p (Assay ID 002299), hsa-miR-374a-5p (Assay ID 000563), hsa-miR-21-5p (Assay ID 000397) and hsa-miR-34a-5p (Assay ID 000426). The expression level of each miRNA was evaluated using the comparative threshold cycle method $(\Delta \Delta \mathrm{Ct})$ and normalized with a corresponding miRNA sequence from $C$. elegans as an exogenous normalizer in gene expression (spike-in celmiR-39). The relative concentration of each miRNA was described by the equation $\Delta \mathrm{Ct}=(\mathrm{Ct}$ miRNA-Ct spike $)$. The cut-off value was set as the cycle $\leq 40$ and it was considered that a gene was not detectable when the $\mathrm{Ct}$ was > 40 and the signal was under established limits $[12,13]$.

\section{Protein quantification}

The serum concentration of the Notch1 protein, whose mRNA is the target of miR-34a, was performed using an ELISA kit ( $R$ \& D Systems Human Notch1 DuoSet ELISA), following the manufacturer's instructions.

\section{Statistical analysis}

To obtain the sample size, the free software G Power (version 3.1.9.2; Heinrich-Heine-Universität, Düsseldorf, Germany) was used. According to the results obtained in the screening phase where we found down-regulation of miR-34a, we calculated the sample size from 2 proportions to $30 \%$ between patients with COPD due to biomass and COPD due to tobacco.

The demographic and clinical characteristics of the study populations were expressed as mean \pm SD. The statistical analysis was carried out by means of ANOVA Tukey's posthoc test to multiple comparisons and the differences, while comparison between two groups were determined by Student's t-test. The statistical analysis for qPCR array was perform with the Qiagen software (available at https://www. qiagen.com/us/shop/genes-and-pathways/data-analysis-center-overview-page/). RT-qPCR was analyzed by relative quantification ( $\Delta \Delta \mathrm{Ct}$ method). The differential expression of a miRNA, and the Notch1 protein quantification was also evaluated by Student's t-test. The analyses were performed using the statistical package GraphPad version 6.01 (GraphPad Software, Inc., La Jolla, CA, USA). $P$ values less than 0.05 were considered significant in all cases.

\section{Results}

\section{Patient characteristics}

Table 1 shows the anthropometric, clinical, and physiological characteristics of the groups. $\mathrm{FEV}_{1} \%$ pred, and 
Table 1 Anthropometric, clinical, and physiological characteristics of the study in women. Data are expressed as the mean \pm SD ( $n=25)$

\begin{tabular}{|c|c|c|c|c|c|}
\hline Group & C & COPD-TS & COPD-BS & $\mathrm{H}-\mathrm{TS}$ & $\mathrm{H}-\mathrm{BS}$ \\
\hline \multicolumn{6}{|l|}{ Characteristics } \\
\hline Age (years) & $66.59 \pm 8.11$ & $69.4 \pm 7.09$ & $73.04 \pm 6.66$ & $62.12 \pm 13.18$ & $65.54 \pm 11.52$ \\
\hline Height (cm) & $158 \pm 8.10$ & $153.92 \pm 8.72$ & $147.67 \pm 8.45^{*} / *$ & $160 \pm 5.9$ & $144 \pm 8.60^{*} / *$ \\
\hline Weight (Kg) & $68.55 \pm 11.59$ & $68.8 \pm 11.58$ & $58.10 \pm 12.25$ & $71.77 \pm 19.56$ & $61.62 \pm 12.85$ \\
\hline $\mathrm{BMI}\left(\mathrm{Kg} / \mathrm{m}^{2}\right)$ & $27.33 \pm 3.91$ & $29.21 \pm 5.48$ & $26.73 \pm 5.62$ & $28.86 \pm 5.48$ & $29.63 \pm 5.04$ \\
\hline \multicolumn{6}{|c|}{ Physiological characteristics } \\
\hline $\begin{array}{l}\mathrm{FEV}_{1} \\
\% \text { pred }\end{array}$ & $96.25 \pm 3.1$ & $68.81 \pm 5.26^{*}$ & $71.95 \pm 6.16^{*} / *$ & $86.32 \pm 4.2 /^{*}$ & $80.14 \pm 2.3 /^{*}$ \\
\hline $\mathrm{FEV}_{1} / \mathrm{FVC}$ ratio & $80.02 \pm 2.4$ & $58.13 \pm 3.1$ & $59.12 \pm 4.0$ & $74.12 \pm 2.3$ & $74.5 \pm 2.2^{*} / *$ \\
\hline GOLD grades & Case numbers (\%) & & & & \\
\hline I & 0 & $4(16)$ & $4(16)$ & 0 & 0 \\
\hline$\|$ & 0 & $21(84)$ & $21(84)$ & 0 & 0 \\
\hline
\end{tabular}

Abbreviations: $B M I$ body mass index, $C$ control healthy women, COPD-BS COPD secondary to biomass smoke exposure, COPD-TS COPD secondary to tobacco smoking, FEV1\% pred forced expiratory volume in 1 second (\% predicted), FVC forced vital capacity, H-BS exposed to BS without COPD, H-TS exposed to TS without COPD. Data were analysed by a one-way ANOVA and Tukey's post hoc test. ${ }^{*} P<0.01$; ${ }^{*}$ vs control; ${ }^{*}$ vs COPD-TS, H-TS or H-BS groups, respectively

the $\mathrm{FEV}_{1} / \mathrm{FVC}$ ratio in both groups of women with COPD showed differences when compared with the $\mathrm{H}-$ BS, H-TS and C groups $(P<0.01)$ with no difference between the groups with COPD. Women with COPD-TS, COPD-BS, H-TS and H-BS were shorter than the $\mathrm{C}$ $(P<0.01)$. The average exposure to $\mathrm{BS}$ in the COPD-BS group was $361 \pm 177 \mathrm{~h} /$ year, while in the COPD-TS group; there was an average cumulative tobacco consumption of $36 \pm 23$ packs/year.

Differential expression of miRNAs in serum by PCR arrays The analyses of expression of miRNAs were performed on samples from 3 women chosen in a simple random way in each study group. Six miRNAs were differentially expressed; 3 were up-regulation, miR-150-5p, miR-191$5 p$ and miR-223-3p, in the COPD-BS group compared with the H-BS group, and the remaining 3 were downregulated, miR-374a-5p in the COPD-BS group compared with $\mathrm{C}$, miR-21-5p in the COPD-TS group compared with $\mathrm{C}$, and miR-34a-5p which was downregulated in the COPD-BS group compared with the COPD-TS group (Table 2).

\section{Validation of miRNAs by RT-qPCR}

To validate the differentially expressed miRNAs obtained in the PCR matrices $(n=3)$, the cDNAs were obtained using the RT kit and TaqMan Universal Master Mix II with UNG (Applied BiosystemsThermo Fisher Scientific). The six miRNAs validated by RT-qPCR $(n=25)$ were as follows: miRNA-34a-5p was down-regulated in the COPD-BS compared with the COPD-TS group (Fig. 2; $P<0.001$ ), miR-374a-5p was down-regulated in the COPD-BS compared with the controls (Fig. 3; $P<0.001$ ), miR-150-5p that was down-regulated in the PCR array analysis did not correspond with the study being decreased by RT-qPCR (Fig. 4a; $P<0.01$ ). The same result was observed with miR-223-3p (Fig. 4b; $P<0.001$ ), when comparing women from the COPD-BS group with those from the H-BS group, while miR-191-5p corresponded with the PCR result and was up-regulated in the COPD-BS group compared with the H-BS group (Fig. 4c; $P<$ 0.01 ), and miR-21-5p was down-regulated in the COPD-TS group compared with the controls (Fig. 5; $P<0.05)$.

Table 2 miRNA differentially expressed in the serum of women in the COPD-BS, COPD-TS (GOLD stages I-II), H-BS and C groups

\begin{tabular}{|c|c|c|c|c|}
\hline Compared groups & microRNA & $\begin{array}{l}\text { Regulation } \\
\text { level }\end{array}$ & Fold change & $P$-value \\
\hline COPD-BS vs. COPD-TS & hsa-miR-34a-5p & down-regulated & -8.77 & 0.020460 \\
\hline COPD-BS vs. C & hsa-miR-374a-5p & down-regulated & -12.29 & 0.000291 \\
\hline \multirow[t]{3}{*}{ COPD-BS vs. H-BS } & hsa-miR-150-5p & up-regulated & 20.66 & 0.000064 \\
\hline & hsa-miR-191-5p & up-regulated & 24.95 & 0.004144 \\
\hline & hsa-miR-223-3p & up-regulated & 30.19 & 0.000645 \\
\hline COPD-TS vs. C & hsa-miR-21-5p & down-regulated & -17.89 & 0.007987 \\
\hline
\end{tabular}

Data are expressed as the regulation, fold change and P-value

Abbreviations: $C$ control healthy women, COPD-BS COPD by biomass smoke exposure, COPD-TS COPD by tobacco smoke, $H-B S$ women exposed to BS without COPD 


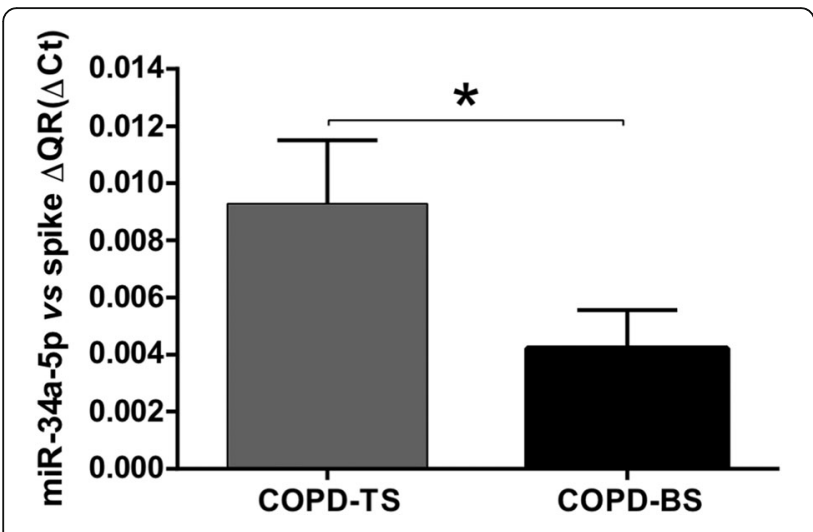

Fig. 2 miR-34a-5p is down-regulated in COPD-BS compared COPDTS. RT-qPCR analysis of serum miR-34a-5p in women with COPD-TS compared with those with COPD-TS and presented as $\Delta \mathrm{Ct}$ values. $n=25$. A statistical difference was observed between women with COPD-BS and women with COPD-TS. Student's t-test was used. * $P<0.001$. Abbreviations: COPD-TS, COPD due to tobacco smoke; COPD-BS, COPD due to biomass smoke.

\section{Serum Notch1 concentration}

To interpret the possible biological relevance of the detected miRNAs in the pathogenesis of COPD, an analysis of the targets of miR-34a-5p was performed specifically because of the subexpression observed in the COPD-BS group compared with the COPD-TS group.

The target was searched in the updated database DIANA TOOLS, miRTarBase-bio.tools and TargetScan. The investigated focused on the participation of miR-34a in COPD-TS and other pulmonary diseases resulting in the Notch1 protein. The serum concentration of Notch1 was quantified by ELISA and was elevated in women in the COPD-BS group compared with women in the COPD-TS group (Fig. 6; $P<0.001$ ) and exhibited an inverse association with the expression of miR-34a-5p.

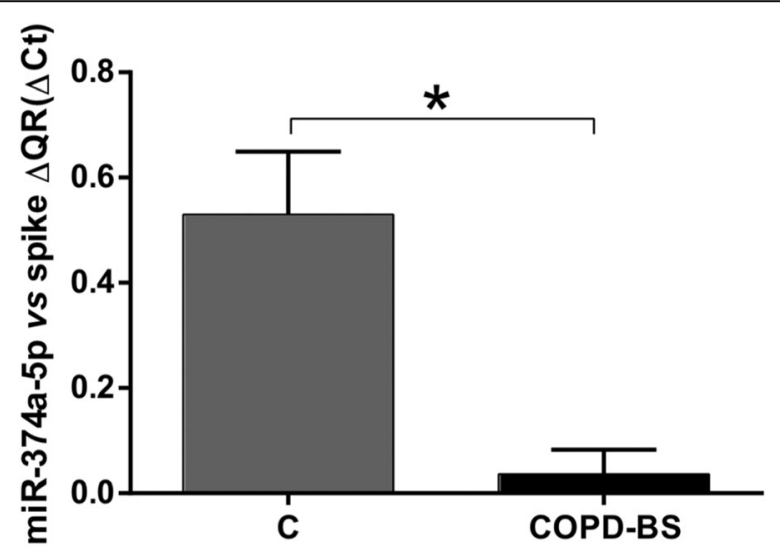

Fig. 3 miR-374-5p is down-regulated in COPD-BS compared with C. RT-qPCR analysis of serum miR-374-5p in women with COPD-BS compared with $C ; n=25$. The data are presented as $\Delta C t$ values. Student's t-test was used. COPD-BS, COPD due to biomass smoke; C, control women. * $P<0.001$

\section{Discussion}

We aimed to analyze the differential expression of miRNAs across five groups of participants with and without COPD by tobacco and biomass exposure. The main finding was that miR-34a down-regulated was differentially expressed between COPD-TS and COPD-BS. We detected differences in 5 miRNAs, miR-374a miR-1915p, miR-21-5p, miR-150, miR-223, yet, these differences were not statistically different between COPD-TS and COPD-BS.

A differential expression of miR-34 in TS-COPD compared to healthy subjects has been reported; however, in the TS-COPD case miR-34 was up-regulated, with a consequent activation of $\mathrm{p} 53$. The expression of miR-34 has been also linked to the severity of TS-COPD, suggesting that miR-34a contributes to the pathogenesis of COPD, by activation in the HIF- $1 \alpha$ pathway (hypoxia-inducible factor) [14]. Another study reported that miR$34 \mathrm{a}$ activation is induced by oxidative stress through PI3K (phosphoinositide-3-kinase) signaling, and it is implicated in aging responses to oxidative stress; thus, miR-34a could become a new therapeutic target and biomarker in COPD and age-related diseases driven by oxidative stress [15]. Contrary to the up-regulated of miR34a in COPD-TS, our results in COPD-BS are down-regulated, which probably gives us the preliminary basis for inferring that miR-34a could distinguish the genotypic characteristics of COPD-BS patients with respect to COPD-TS patients.

To understand one of the possible biological implications of the down-regulation of miR-34a in COPD-BS, one of its targets, the Notch1, was selected. miR-34a reduces the action of the Notch1 pathway, which plays an important role in the differentiation of the epithelium in the human airway. It has been observed that the addition of a Notch1 ligand, or the constitutive expression of its receptor, increases the number of mucosal cells containing MUC5AC and the number of secretory cells $[16,17]$. Focusing on our findings, we can infer that patients with COPD by biomass, which have miR-34a down-regulated, do not supress the activation of Notch1 signaling, increasing the number of secretory cells, as has been shown in in vitro studies [17]. Our finding provides a potential explanation for the chronic bronchitis clinical expression of COPD-BS, likely mediated by the downregulation of miR-34a. This is relevant, because the regulation of Notch 1 could represent an important therapeutic target for these patients.

Another interesting finding of our study is the differential expression of three miRNAs between the study groups: miR-374a down-regulated in the group $\mathrm{C}$ vs COPD-BS, miR-191 up-regulated in the group $\mathrm{H}$ vs COPD-BS and miR-21 down-regulated in the group $C$ vs COPD-TS. The miR-374a has been reported to be 


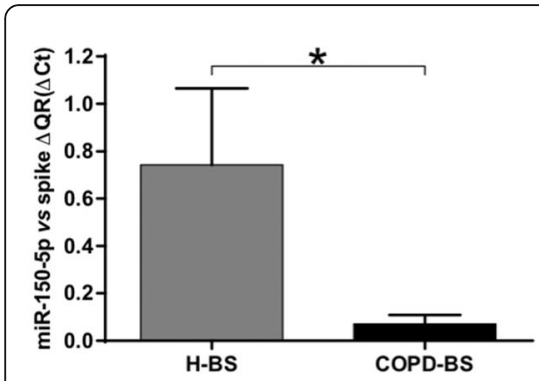

a

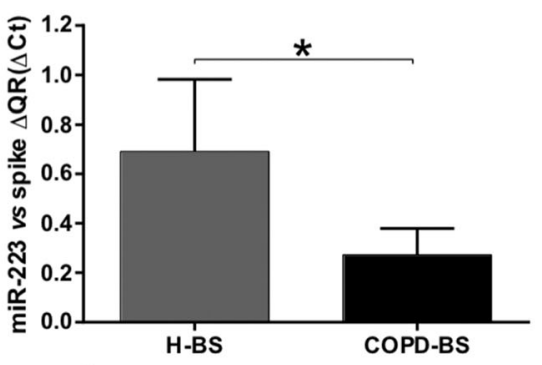

b

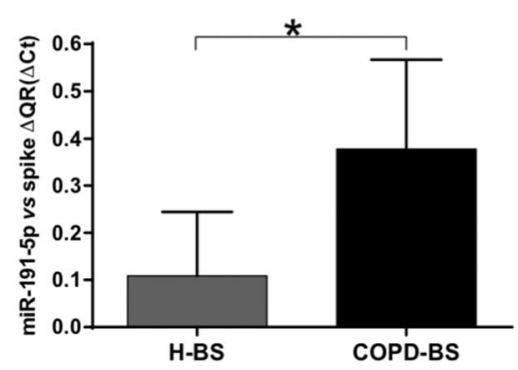

C

Fig. 4 miR-150-5p and miR-223-3p are down-regulated and miR-191-5p is up-regulated in women with COPD-BS compared with H-BS. RT-qPCR analysis of serum miR-150-5p (a), miR-223-3p (b), and miR-191-5p (c) in women with COPD-BS compared with H-BS; $n=25$. The data are presented as $\Delta C t$ values. Student's t-test was used. COPD-BS, COPD due to biomass smoke; H-BS, women exposed to BS without COPD. ${ }^{* *} P<0.01,{ }^{*} P<0.001$

up-regulated in skeletal muscle in patients with COPDTS, and associated with the development of extrapulmonary manifestations and co-morbidities in COPD-TS [18]. Base on the findings we suggest that it could be a good indicator of the comorbidities of COPD-BS patients, although more in-depth studies are needed to determine this possibility. miR-191, has been reported to be up-regulated in lung tissue and bronchoalveolar lavage (BAL) of mice exposed to TS, associated with the development of inflammatory cells in lung and lung parenchyma [19]. The results suggest that COPD due to tobacco and biomass could share the same pathway of inflammation; however, our results need to be evaluated with more studies. Another miRNA validated was miR21 , this miR in COPD-TS has been reported as upregulated in asymptomatic smokers [20]. Another study demonstrated that up-regulated of miR-21 in plasma and mononuclear cells of patients with COPD-TS may contribute to their pathogenesis and severity [21],

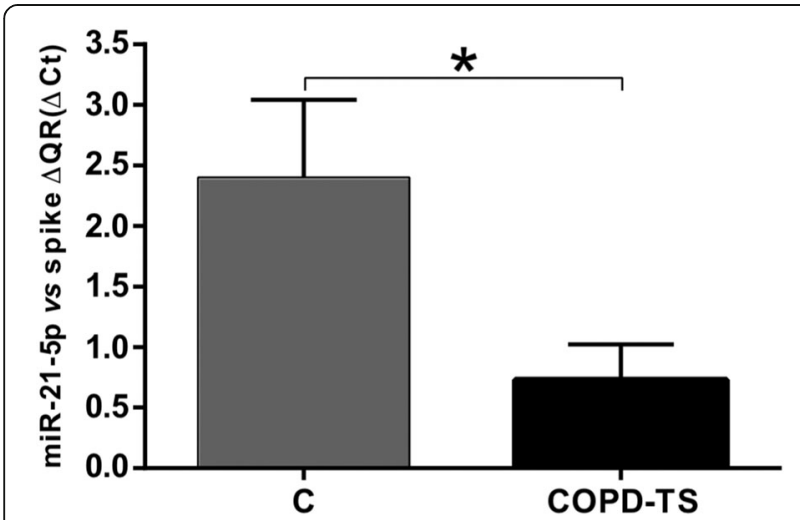

Fig. 5 miR-21-5p is down-regulated in women with COPD-TS compared with C. RT-qPCR analysis of serum miR-21-5p in women with COPD-TS compared with $C ; n=25$. The data are presented as $\Delta \mathrm{Ct}$ values. Student's t-test was used. COPD-TS, COPD due to tobacco smoke; C, control women. ${ }^{* *} P<0.05$ suggesting that high serum plasma levels of miR-21 may be a diagnostic and therapeutic indicator in COPD-TS [20], so that, our results were consistent with previous studies.

\section{Limitations of the study}

The limitations of this study are related to its sample size. We used a small number of participants in the screening phase, to identify key miRNAs to be later validated in the total sample. This procedure will lead to the identification of miRNAs that are very different across groups but will fail to identify miRNAs that are more similar, which could cloud our understanding of partially expressed miRNAs. For this purpose, a larger sample size is needed. Additionally, only Notch1 was quantified, one of the many targets of miR-34a. Still, this limited

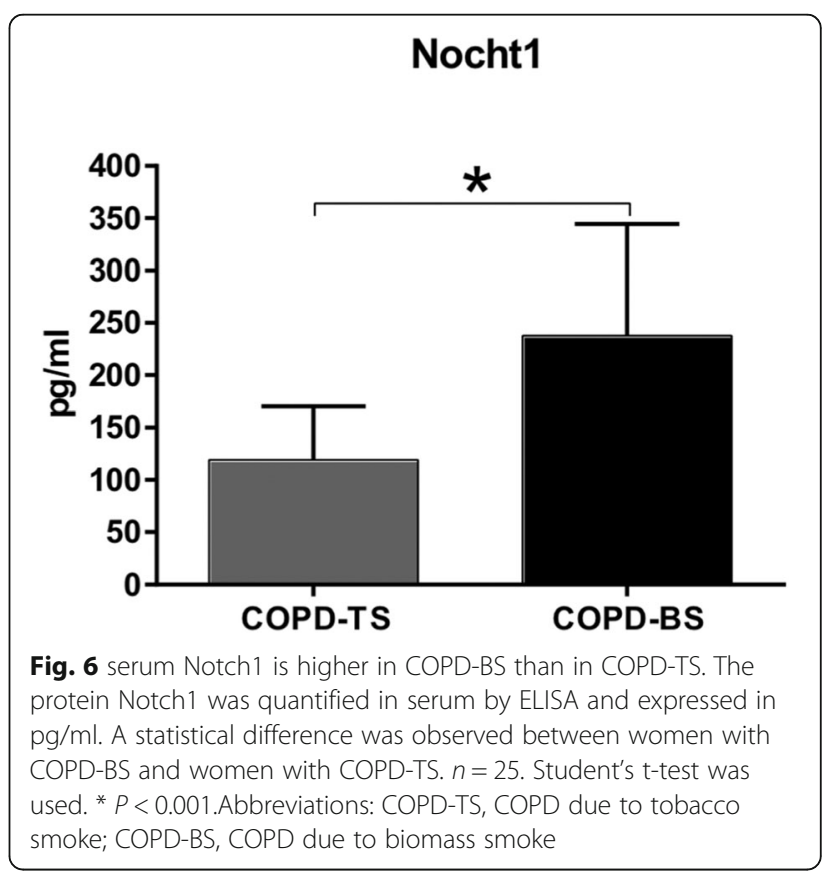


analysis allowed us to begin to understand the relevance of miRNAs in COPD-BS, considering the great complexity of this disease. Coupled with this, characteristics such as the socio-economic level, the level of education, ethnic origin, genetic susceptibility and various other environmental factors, as well as the type and severity of exposure to BS or TS in the study groups could influence our results.

\section{Conclusions}

This is the first study in patients with COPD due to biomass that demonstrates the genotypic difference between patients determined by miRNAs, supporting that COPD by biomass has a different genotype than COPD due to tobacco, which could have important implications for the treatment of these diseases.

\section{Abbreviations}

BMI: Body mass index; BS: Biomass smoke exposure; C: Control healthy women; COPD-BS: COPD by biomass smoke exposure; COPD-TS: COPD by tobacco smoking; FEV $\%$ pred: forced expiratory volume in the 1 st sec (\% predicted); FVC\% pred: forced vital capacity (\% predicted); GOLD: Global Initiative for Chronic Obstructive Lung Disease; H-BS: Women exposed to BS without COPD; H-TS: Smokers without COPD; miR: microRNA miRNAs: MicroRNAs; RT-qPCR: Real-time reverse transcriptionase-PCR; TS: Tobacco smoking

\section{Acknowledgements}

We are grateful to PhD. Eduardo Montes Martinez at Clinica of Asthma), and to MSc. Christian Adolfo Trejo Jasso for his valuable advice and help in the development of RT-qPCR and ELISA techniques at National Institute of Respiratory Diseases Ismael Cosio Villegas (INER).

\section{Authors' contributions}

YVT, VRL, OPB, CR, and MM made substantial contributions to conception and design. YVT, VRL, IBR, OPB, ARV, JPR, CR, and MM made acquisition of data. $Y V T, V R L, O P B, R F V, C R$, and $M M$ made analysis and interpretation of data. YVT, VRL, OPB, RFV, CR, and MM have been involved in drafting the manuscript. $\mathrm{VT}, \mathrm{VRL}, \mathrm{OPB}, \mathrm{RFV}, \mathrm{CR}$, and $\mathrm{MM}$ have been involved in revising it critically for important intellectual content. All authors have given final approval of the version to be published and agreed to be accountable for all aspects of the work

\section{Funding}

This research was support by the Consejo Nacional de Ciencia y Tecnología (CONACyT), Mexico; grant number: FOSISS; SALUD-2016-1-272301. The role of the funding of this study included the acquisition of reactive, consumables, laboratory equipment and all necessary to develop the research, CONACyT did not participate directly in the collection, analysis and interpretation of data, nor in the writing of the manuscript.

\section{Availability of data and materials}

The datasets generated during and/or analyzed during the current study are available from the corresponding author on reasonable request.

\section{Ethics approval and consent to participate}

The Science, Bioethics and Biosafety Committees of the National Institute of Respiratory Diseases Ismael Cosío Villegas approved the study (INER) in Mexico City. All participants of the study were recruited at the COPD Clinic of INER, a signed informed consent form was obtained from all participants. The research was developed according with the Official Mexican Standard NOM-012-SSA3-2012, which establishes the criteria for the execution of research projects for human health. The protocol approved at INER was the B15 15.
Consent for publication

Not applicable

\section{Competing interests}

The authors declare that they have no competing interests.

\section{Author details}

${ }^{1}$ Department of Biological Systems, Autonomous Metropolitan

University-Xochimilco (UAM-X), Mexico City, Mexico. ${ }^{2}$ Biological and Health Sciences, Autonomous Metropolitan University-Xochimilco (UAM-X), Mexico City, Mexico. ${ }^{3}$ Clinic of Smoking and COPD, Mexico City, Mexico. ${ }^{4}$ Laboratory of Molecular Biology, Mexico City, Mexico. ${ }^{5}$ Laboratory of Translational Research in Aging and Pulmonary Fibrosis, Mexico City, Mexico. ${ }^{6}$ Laboratory of Cell Biology, Department of Research in Pulmonary Fibrosis, Mexico City, Mexico. ${ }^{7}$ Laboratory of HLA, National Institute of Respiratory Diseases Ismael Cosio Villegas (INER), Calzada de Tlalpan 4502, Col Section XVI, C.P. 14080

Tlalpan, Mexico City, Mexico.

Received: 15 March 2019 Accepted: 31 October 2019

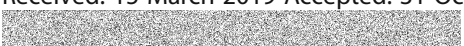

\section{References}

1. Global Initiative for Chronic Obstructive Lung Disease (GOLD) 2019. Available from: GOLD-2019-POCKET-GUIDE-FINAL_WMS.pdf

2. Ramírez-Venegas A, Sansores RH, Pérez-Padilla R, Regalado J, Velázquez A, Sánchez C, et al. Survival of patients with chronic obstructive pulmonary disease due to biomass smoke and tobacco. Am J Resp Crit Care Med. 2006;173:393-7.

3. Golpe R, Sanjuán López P, Cano Jiménez E, Castro Añón O, Pérez de Llano LA. Distribution of Clinical Phenotypes in Patients with Chronic Obstructive Pulmonary Disease Caused by Biomass and Tobacco Smoke. Arch Bronconeumol. 2013;50:318-24.

4. Ramirez A, Sansores R, Velazquez M, Perez O. Nonsmoker and biomass exposure, in Controversies in COPD. In: Anzueto A, Heijdra Y, Hurst JR, editors. 2015, European Respiratory Society monograph. UK; 2015. p. 35-45. Recovered from the site https://books.ersjournals.com/content/controversies-in-copd.

5. Sood A, Assad NA, Barnes PJ, Churg A, Gordon SB, Harrod KS, et al. ERS/ATS workshop report on respiratory health effects of household air pollution. Eur Respir J. 2018:51:1700698.

6. Ramírez-Venegas A, Velázquez-Uncal M, Pérez-Hernández R, Guzmán-Bouilloud NE, Falfán-Valencia R, Mayar-Maya ME, Aranda-Chávez A, Sansores RH. Prevalence of COPD and respiratory symptoms associated with biomass smoke exposure in a suburban area. Int J Chron Obstruct Pulmon Dis. 2018;13:1727-34.

7. Mitchell PS, Parkin RK, Kroh EM, Fritz BR, Wyman SK, Pogosova-Agadjanyan EL, et al. Circulating microRNAs as stable blood-based markers for cancer detection. Proc Natl Acad Sci U S A. 2008:105:10513-8.

8. Akbas F, Coskunpinar E, Aynaci E, Oltulu YM, Yildiz P. Analysis of serum micro-RNAs as potential biomarker in chronic obstructive pulmonary disease. Exp Lung Res. 2012;38:286-94.

9. Ruiz-Vera T, Ochoa-Martínez ÁC, Pruneda-Álvarez LG, Zarazúa S, PérezMaldonado IN. Exposure to biomass smoke is associated with an increased expression of circulating miRNA-126 and miRNA-155 in Mexican women: a pilot study. Drug Chem Toxicol. 2019;42:335-42.

10. Pérez-Padilla JR, Regalado-Pineda J, Vázquez-García JC. Reproducibility of spirometry in Mexican workers and international reference values. Salud Publica de Mex. 2001;43:113-21.

11. Hankinson JL, Odencrantz JR, Fedan KB. Spirometric reference values rom a sample of the general U.S. population. Am J Respir Crit Care Med. 1999;159: 179-87.

12. Marabita F, de Candia P, Torri A, Tegnér J, Abrignani S, Rossi RL. Normalization of circulating microRNA expression data obtained by quantitative real-time RT-PCR. Brief Bioinform. 2015;17:204-12.

13. Faraldi M, Gomarasca M, Sansoni V, Perego S, Banfi G, Lombardi G. Normalization strategies differently affect circulating miRNA profile associated with the training status. Sci Rep. 2019;9:1584.

14. Cui H, Ge J, Xie N, Banerjee S, Zhou Y, Antony VB, Thannickal VJ, Liu G. miR$34 \mathrm{a}$ inhibits lung fibrosis by inducing lung fibroblast senescence. Am J Respir Cell Mol Biol. 2017:56:168-78.

15. Chang TC, Wentzel EA, Kent OA, Ramachandran K, Mullendore M, Lee KH, et al. Transactivation of miR-34a by p53 broadly influences gene expression and promotes apoptosis. Mol Cell. 2007;26:745-52. 
16. Tilley AE, Harvey BG, Heguy A, Hackett NR, Wang R, O'Connor TP, et al. Down-regulation of the notch pathway in human airway epithelium in association with smoking and chronic obstructive pulmonary disease. Am J Respir Crit Care Med. 2009;179:457-66.

17. Zong D, Ouyang R, Li J, Chen Y, Chen P. Notch signaling in lung diseases: focus on Notch1 and Notch3. Ther Adv Respir Dis. 2016;10:468-84.

18. Võsa U, Vooder T, Kolde R, Fischer K, Välk K, Tönisson N, et al. Identification of miR-374a as a prognostic marker for survival in patients with early-stage nonsmall cell lung cancer. Genes Chromosomes Cancer. 2011;50:812-22.

19. Conickx G, Avila Cobos F, van den Berge M, Faiz A, Timens W, Hiemstra PS, et al. microRNA profiling in lung tissue and bronchoalveolar lavage of cigarette smoke-exposed mice and in COPD patients: a translational approach. Scientific Reports. 2014;7:12871.

20. Xie L, Wu M, Lin H, Liu C, Yang H, Zhan J, et al. An increased ratio of serum miR-21 to miR-181a levels is associated with the early pathogenic process of chronic obstructive pulmonary disease in asymptomatic heavy smokers. Mol BioSyst. 2014;10:1072-81.

21. Xie L, Yang F, Sun S. Expression of miR-21 in peripheral blood serum and mononuclear cells in patients with chronic obstructive pulmonary disease and its clinical significance. Zhong Nan Da Xue Xue Bao Yi Xue Ban. 2016; 41:238-43.

\section{Publisher's Note}

Springer Nature remains neutral with regard to jurisdictional claims in published maps and institutional affiliations.

Ready to submit your research? Choose BMC and benefit from:

- fast, convenient online submission

- thorough peer review by experienced researchers in your field

- rapid publication on acceptance

- support for research data, including large and complex data types

- gold Open Access which fosters wider collaboration and increased citations

- maximum visibility for your research: over $100 \mathrm{M}$ website views per year

At $\mathrm{BMC}$, research is always in progress.

Learn more biomedcentral.com/submissions 